\title{
Joint Relay Selection and Link Adaptation for Distributed Beamforming in Regenerative Cooperative Networks
}

\author{
Wei Yang*, Lihua $\mathrm{Li}^{*}$, Gang $\mathrm{Wu}^{\dagger}$, Haifeng Wang ${ }^{\dagger}$ \\ ${ }^{*}$ Key Lab. of Universal Wireless Commun., Beijing University of Posts and Telecom.(BUPT) \\ Ministry of Education, Wireless Technology Innovation Institute(WTI), BUPT, China \\ ${ }^{\dagger}$ Wireless Modem System Research, Device R\&D, NOKIA, Shanghai, China
}

\begin{abstract}
Relay selection enhances the performance of the cooperative networks by selecting the links with higher capacity. Meanwhile link adaptation improves the spectral efficiency of wireless data-centric networks through adapting the modulation and coding schemes (MCS) to the current link condition. In this paper, relay selection is combined with link adaptation for distributed beamforming in a two-hop regenerative cooperative system. A novel signaling mechanism and related optimal algorithms are proposed for joint relay selection and link adaptation. In the proposed scheme, there is no need to feedback the relay selection results to each relay. Instead, by broadcasting the link adaptation results from the destination, each relay will automatically understand whether it is selected or not. The lower and upper bounds of the throughput of the proposed scheme are derived. The analysis and simulation results indicate that the proposed scheme provides synergistic gains compared to the pure relay selection and link adaptation schemes.
\end{abstract}

Index Terms-relay selection, link adaptation, distributed beamforming, signaling, throughput.

\section{INTRODUCTION}

Cooperative communication networks, in which wireless nodes cooperate with each other in transmitting information, promise significant gains in overall throughput and create robustness against channel fading [1] [2]. A variety of cooperative schemes have been proposed in the literatures with different design issues and channel information assumptions.

Link adaptation can help mitigate the effects of time-varying fading channel as well as exploit favorable channel conditions when they exist [3]. Link adaptation for cooperative networks are considered in [4] to maximize the data throughput.

Independent of link adaptation, the network performance can also be improved by selecting the cooperating relays to exploit temporal and spatial diversity [5]. Relay selection simplifies signaling, avoids complex synchronization schemes, and with careful design can preserve the spatial diversity provided by the total number of relays in the network [6].

For the networks with parallel relays, distributed beamforming (also called network beamforming in [7]) is proved optimal if perfect channel state information (CSI) or high quality

This paper is supported by the National Science Foundation of China (NSFC 60702051, NSFC-AF: 60910160), the Specialized Research Fund for the Doctoral Program of Higher Education (SRFDP£20070013028), and the Program for New Century Excellent Talents in University (NCET-08-0735). This paper is co-funded by Nokia on the Beyond 3G Research project. channel information feedback from the receiver is available at the transmitter [8]. In this approach, the relays linearly weight their transmit signals according to the CSI so that they can add up coherently at the destination. The beamforming problem for networks with limited feedback from destination to relays is studied in [9] for non-regenerative relays, and in [10] for regenerative relays.

The combination of relay selection and link adaptation is proposed in [11]. However, only single relay is selected, and distributed beamforming is not considered in this scheme. Moreover, it is designed for multi-hop transmission, in which relay and modulation and coding scheme (MCS) are selected in each hop by the forwarding relays (acting as the source in each hop).

In this paper, joint relay selection and link adaptation are considered for two-hop regenerative cooperative systems. The modulation scheme and relays are selected for distributed beamforming to forward data from the source to the destination. Unlike that in [11], the selection in our proposal is performed by the destination, and the selection results are fed back to the source and relays. To reduce the signaling overhead of the relay selection and link adaptation, a novel signaling mechanism is then proposed to broadcast the link adaptation results from the destination, and each relay will automatically understand whether it is selected or not. Moreover, a simple relay and modulation scheme selection algorithm is designed with the complexity linear to the number of candidate modulation schemes. The analysis and simulation results of the throughput and symbol error rate (SER) show that the proposed scheme outperforms pure relay selection and link adaptation schemes.

This paper is organized as follows. Section $\amalg$ presents the system model. Section $\Pi$ iII introduces the joint relay selection and link adaptation scheme, and the optimal selection algorithm. The lower and upper bounds of the throughput of the proposed scheme are derived in Section IV and simulation results of the throughput and SER performance are provided. Finally, conclusions are drawn in Section $\mathrm{V}$

\section{System Model}

Consider a two-hop regenerative cooperative system with a source node, a destination node and a set of $N$ candidate 


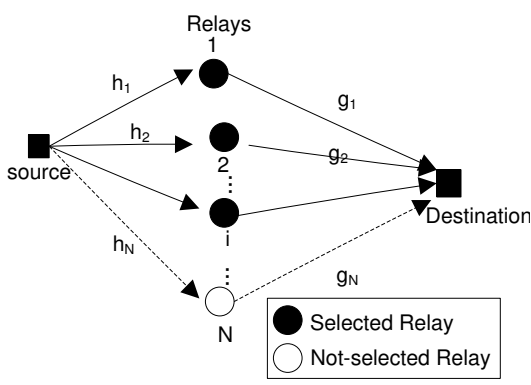

Fig. 1. Wireless network with parallel relays.

relays $\mathcal{R}=\{1,2, \cdots N\}$, as illustrated in Fig 11 Each relay node has only one antenna which cannot transmit and receive simultaneously. Assume there is no direct link between the source and the destination. Denote the channel from the source to the $i$-th relay as $h_{i}$ and the channel from the $i$-th relay to the destination as $g_{i} . h_{i}$ and $g_{i}$ are assumed to be independent and modeled as $C \mathcal{N}(0,1)$. The received noise at each receiver is normally distributed $\sim \mathrm{CN}\left(0, N_{0}\right)$. The source transmits under an average power constraint $P_{s}$, and relays are under a total power constraint, which is equal to $P_{s}$. Denote $\rho$ as the signalto-noise ratio (SNR) without fading, i.e., $\rho=\frac{P_{s}}{N_{0}}$. Depending on the channel states, only a subset, $\mathcal{A} \subset \mathcal{R}$ is selected to help the transmission between the source and the destination. $A$ is used to denote the cardinality of $\mathcal{A}$.

In the first hop, the source node transmits $s$ to the relays, where $s \in C$, and $C$ is a finite constellation with average unit energy and $M$ cardinality. For simplicity of the performance analysis, square $M$-ary quadrature amplitude modulation ( $M$ QAM) is used as candidate modulation scheme (MS). However, the proposed scheme do not limit to this assumption, and continue to work in a coded system with various MCS. Denote QAM scheme with $2^{2 k}$ cardinality as $M S_{(k)}, k=1, \cdots L$, and $\mathcal{S}=\left\{M S_{(1)}, M S_{(2)}, \ldots M S_{(L)}\right\}$ as the set of MSs. The $i$ th relay receives

$$
r_{i}=\sqrt{P_{s}} h_{i} s+n_{i}
$$

where $P_{s}$ is the average power used at the source, and $n_{i}$ is the noise at relay node $i$. The $i$-th relay demodulates the received signal as $\hat{s}_{i}$ using maximum likelihood (ML) demodulation,

$$
\hat{s}_{i}=\arg \min _{s_{i} \in \mathcal{C}}\left\|r_{i}-\sqrt{P_{s}} h_{i} s\right\| .
$$

In the second hop, the selected relays transmit simultaneously to the destination using distributed beamforming. The beamforming weight for the $i$-th selected relay is $\omega_{i}, i \in \mathcal{A}$. Assume the MS used in the second hop is the same as the first hop. ${ }^{1}$ The received signal at the destination is

$$
y_{d}=\sum_{i \in \mathcal{A}} \sqrt{P_{s}} g_{i} \omega_{i} \hat{s}_{i}+n_{d}
$$

where $n_{d}$ is the noise at the destination.

\footnotetext{
${ }^{1}$ Fixing modulation scheme simplifies the design of relays and avoids complex signaling during the data transmission, although using a different modulation schemes for the second phase transmission can bring extra throughput gains [2].
}

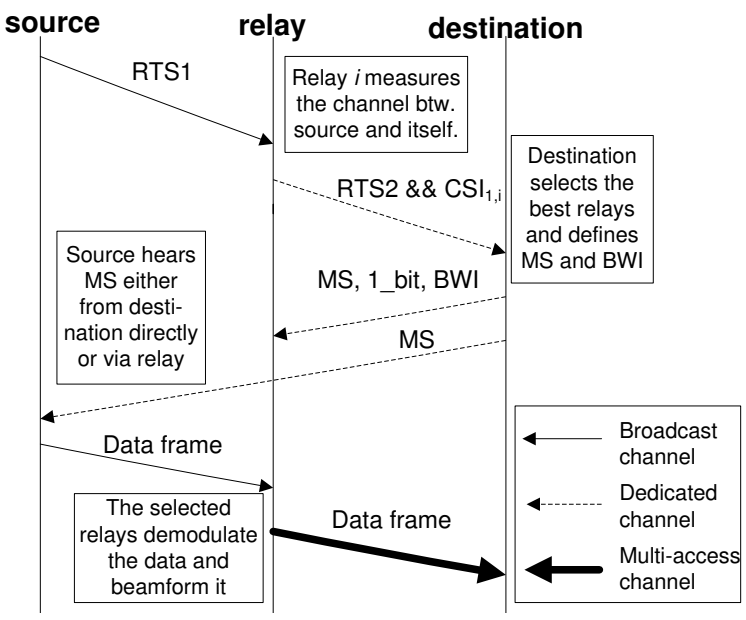

Fig. 2. Flowchart of the proposed general joint relay selection and link adaptation scheme

Given the knowledge of CSI, if all the selected relays demodulate the signal from the source correctly, the optimal beamforming weight at each selected relay $i$ is $\frac{g_{i}^{*}}{\sum_{i \in \mathcal{A}}\left|g_{i}\right|^{2}}$ [2], where $g_{i}^{*}$ is the conjugate value of $g_{i}$.

\section{Joint Relay Selection and Link Adaptation for Distributed BEAMFORMing}

\section{A. The general scheme}

This section presents a scheme for joint relay selection and link adaptation in a regenerative cooperative network, the flowchart of which is shown in Fig. 2

The details of the proposed scheme are described as follows.

Step 1: The source broadcasts a ready-to-send (RTS) packet, denoted as RTS1. Each relay measures the CSI between source and itself upon reception of RTS1, denoted as $C S I_{1, i}$.

Step 2: Relays transmit the RTS2 packets containing $C S I_{1, i}$ to the destination in their dedicated channels.

Step 3: The destination selects the best relays based on $\left|h_{i}\right|^{2}$ and $\left|g_{i}\right|^{2}$. It also decides the MS for the transmission. Here the Best Throughput Criterion is used

$$
\left[M S^{*}, \mathcal{A}^{*}\right]=\arg \max _{M S, \mathcal{A}}\left\{\mathscr{R}(M S) \mid P_{e}\left(M S, \gamma_{d}\right) \leq S E R_{t g t}\right\},
$$

where $\mathscr{R}(M S)$ is the data rate of the corresponding modulation scheme, $\gamma_{d}$ is the instantaneous received SNR at the destination, $P_{e}\left(M S, \gamma_{d}\right)$ is the SER when the corresponding MS is used, and $S E R_{t g t}$ is the target SER. In the following text, we denote $M S_{i}>M S_{j}$, if $\mathscr{R}\left\{M S_{i}\right\}>\mathscr{R}\left\{M S_{j}\right\}$. The Best Throughput Criterion allows us to select the optimal MS and relays, such that the throughput of the system is maximized while the SER is below a certain target.

Step 4: The destination feeds back the selected MS, the relay selection result ( 1 bit to indicate each specific relay whether it is selected or not, denoted as 1_bit in Fig. (2) and the beamforming weight information (BWI) to each relay in its dedicated channel. The source should be also notified the MS either from the destination directly or via the selected relays. BWI is the weight information to perform 


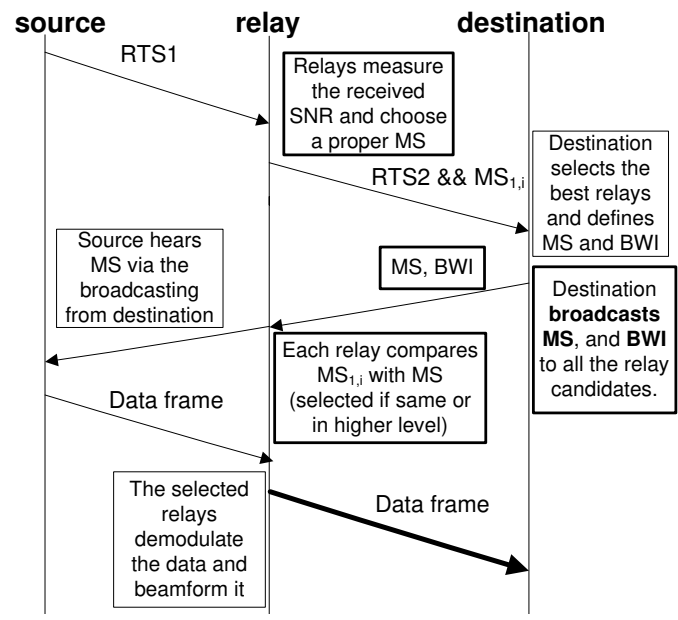

Fig. 3. Flowchart of the proposed broadcasting signaling scheme (vs. the general signaling scheme feedbacks MS, BWI and Relay Selection Index via dedicated channel).

distributed beamforming at each relay. Different BWI feedback mechanisms should be adopted in TDD and FDD systems.

In TDD systems, the destination sends training sequence to relays. The selected relays can estimate the channel gains $\left|g_{i}\right|$ based on channel reciprocity. The destination only needs to broadcast the summation of the channel power gains of the selected relays, $\sum_{i \in \mathcal{A}}\left|g_{i}\right|^{2}$ to the selected relays. Each relay can adjust its transmission power into $\frac{\left|g_{[i]}\right|^{2} P_{s}}{\left(\sum_{i \in \mathcal{A}}\left|g_{[i]}\right|^{2}\right)^{2}}$ accordingly. In FDD system, the destination has to feedback BWI $\frac{g_{i}^{*}}{\sum_{i \in \mathcal{A}}\left|g_{[i]}\right|^{2}}$ to each selected relay.

Step 5: The source broadcasts data using the selected MS.

Step 6: The selected relays demodulate the received signal from the source and beamform it to the destination.

\section{B. Improved feedback signaling}

Both relay selection and link adaptation require some information exchange among source, relays and destination, which is a heavy burden on signaling. Such as in IEEE 802.16j mobile relay (MR) systems, the feedbacks from destination to relays are in each relay's dedicated channel independently, and the feedback channel is pre-scheduled by the scheduler in higher layer before relay selection and link adaptation. Prior to scheduling, none of the nodes know what relays will be actually selected. So the dedicated signaling channel must be scheduled for all the relays, making the overhead proportional to the number of relays in the network.

To reduce the overhead, an improved feedback signaling for the joint relay selection and link adaptation scheme is proposed. The flowchart of the proposed feedback signaling is shown in Fig 3 The process is described in details as follows:

Step 1: The source broadcasts a RTS1 packet. Upon reception of RTS1, all the relays measure the SNR of the channel between source and itself and define MS as $M S_{1, i}$. The MS selection criterion used in the proposed scheme is

$$
M S_{1, i}=\arg \max _{M S}\left\{\mathscr{R}(M S) \mid P_{e}\left(M S, \gamma_{1, i}\right) \leq S E R_{t g t}\right\},
$$

where $\gamma_{1, i}=\rho\left|h_{i}\right|^{2}$ is the SNR of the first hop at each relay. In practical systems, MS can be determined by a threshold based method, and the threshold between $M S_{(k)}$ and $M S_{(k-1)}$ is

$$
\Gamma\left(M S_{(k)}\right)=\arg \min _{\gamma}\left\{\gamma \mid P e\left(M S_{k}, \gamma\right) \leq S E R_{t g t}\right\}, k=1, \cdots L .
$$

Step 2: Each relay sends its RTS2 packet with $M S_{1, i}$ to the destination.

Step 3: The destination then selects the relays, and determines the MS for the first and second hop and BWI. The selection criterion is the same as (4).

Step 4: The destination broadcasts the following information to the source and relays: MS and BWI. Note that, the destination does not need to feedback to each relay the relay selection results. Instead, upon reception of MS from destination, each relay compares it with $M S_{1, i}$, which is determined in Step 2. The relay is selected if

$$
M S_{1, i} \geq M S^{*},
$$

where $M S^{*}$ is the selected MS. Otherwise, the corresponding relay candidates back off. The source can be notified about MS by listening to the broadcasting from destination.

Step 5: The source broadcasts data.

Step 6: The selected relays demodulate the signal from the source and beamform it to the destination.

Note again in Step 4, the relay can know it is selected if $M S^{*}$ is aligned with the link between it and source so that there is no need for the destination to feedback the relay selection result as in the general scheme. Overall, it can be noticed that the signaling overhead can be considerably reduced by broadcasting instead of dedicated signaling.

\section{The relays and $M S$ selection algorithm}

To find the set $\mathcal{A}^{*}$ and modulation scheme $M S^{*}$ in (4), an exhaustive search would involve over $L \cdot 2^{N}$ cases. The computational complexity of the exhaustive search grows exponentially with the number of relay candidates. Below a simple algorithm to find the optimal set $\mathcal{A}^{*}$ and modulation scheme $M S^{*}$ is proposed as Algorithm 1.

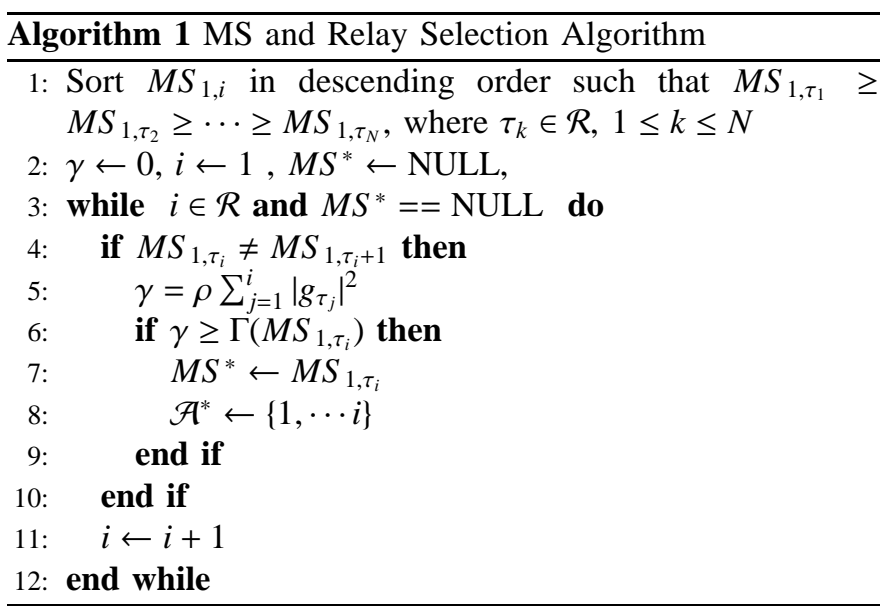

In this algorithm, the selected relays are those which have the largest source-relay channel SNR, among the set of all 


$$
f(u, v)= \begin{cases}\frac{N !}{\rho^{2}(N-A-1) !(A-1) !} e^{-\frac{(u+v)}{\rho}}\left(1-e^{-\frac{v}{\rho}}\right)^{N-A-1} \cdot\left(e^{-\frac{A u}{\rho}} \sum_{j=0}^{A-1} \frac{u^{j}}{\rho^{j} j !}+\frac{u^{A-1}}{\rho^{A-1} A !}\left(e^{-\frac{A u}{\rho}}+e^{-\frac{A v}{\rho}}-1\right)\right), & 0<v<u \\ \frac{N ! u^{A-1}}{\rho^{A+1}(N-A-1) !(A-1) ! A !} e^{-\frac{u}{\rho}} e^{-\frac{(A+1) v}{\rho}}\left(1-e^{-\frac{v}{\rho}}\right)^{N-A-1}, & 0<u<v\end{cases}
$$

candidate relays $\mathcal{R}$. The SNR at the destination when relays in $\left\{1, \cdots \tau_{i}\right\}$ are selected is shown in the line 5 of the Algorithm 1 , assuming no demodulating error at the selected relays. The SNR loss at the destination due to demodulating errors at the selected relays is analyzed in Section [V] It is clear that adding more relays to the set $\mathcal{A}$ increases the SNR at the destination, and hence is always beneficial in the second hop transmission. That explains why the result of MS selection can signal the results of relay selection.

The computational complexity of the proposed algorithm is decreased dramatically. Only less than $L$ cases are involved in addition to a sort operation of $N$ integers.

\section{Performance Evaluation}

\section{A. Performance Analysis}

The instantaneous received SNR at the destination in the second phase can be expressed as,

$$
\begin{aligned}
\gamma_{d} & =\frac{\rho\left|\sum_{i \in \mathcal{A}} \omega_{i} g_{i}\right|^{2}}{1+\rho \sum_{i \in \mathcal{A}} \omega_{i} g_{i} \mathbb{E}\left(\left|\delta_{i}\right|^{2}\right)} \\
& =\frac{\rho \sum_{i \in \mathcal{A}}\left|g_{i}\right|^{2}}{1+\rho / \sum_{i \in \mathcal{A}}\left|g_{i}\right|^{2} \cdot\left(\sum_{i \in \mathcal{A}}\left|g_{i}\right|^{2} \mathbb{E}\left(\left|\delta_{i}\right|^{2}\right)\right)},
\end{aligned}
$$

where $\delta_{i}=\hat{s}_{i}-s$ is the demodulating error of the $i$ th relay. The expectation of the demodulating errors at the $i$ th relay using square MQAM can be approximated as,

$$
\begin{aligned}
\mathbb{E}\left(\left|\delta_{i}\right|^{2}\right) & \approx \frac{4\left(e_{\min }\right)^{2}(\sqrt{M}-1)}{\sqrt{M}} Q\left(\sqrt{\frac{P_{s}\left(e_{\min }\right)^{2}\left|h_{i}\right|^{2}}{2 N_{0}}}\right) \\
& =\frac{24}{M+\sqrt{M}} \cdot Q\left(\sqrt{\frac{3 \gamma_{1, i}}{(M-1)}}\right),
\end{aligned}
$$

where $e_{\min }=\sqrt{\frac{6}{M-1}}$ is the minimum Euclidean distance of MQAM with unit average energy. Substituting (9) into (8), the lower bound of the received SNR can be obtained as

$$
\gamma_{d} \geq \sum_{i \in \mathcal{A}} \gamma_{2, i}-\underbrace{\sum_{i \in \mathcal{A}} \frac{24 \gamma_{2, i}}{M+\sqrt{M}} \cdot Q\left(\sqrt{\frac{3 \gamma_{1, i}}{(M-1)}}\right)}_{\text {SNR loss }}
$$

where $\gamma_{2, i}=\rho\left|g_{i}\right|^{2}$. The second term in (10) is the SNR loss due to the demodulating errors at the selected relays.

The average throughput for the joint link adaptation and relay selection schemes can be defined as

$$
\begin{aligned}
\zeta & =\frac{1}{2} \mathbb{E}[\mathscr{R}(M S)(1-B L E R)] \\
& =\sum_{k=1}^{L} \sum_{\mathcal{A C \mathcal { R }}} k \cdot \operatorname{Pr}\left(\mathcal{A}, M S_{(k)}\right)\left(1-P_{e}\left(\mathcal{A}, M S_{(k)}\right)\right)^{L_{b i t} / 2 k},
\end{aligned}
$$

where $B L E R$ is the block error rate, $\operatorname{Pr}\left(\mathcal{A}, M S_{(k)}\right)$ is the probability that the set $\mathcal{A}$ of relays and modulation scheme $M S_{(k)}$ are selected, $P_{e}\left(\mathcal{A}, M S_{(k)}\right)$ is the SER and $L_{b i t}$ is the length of each transmitted block.

Using symmetry arguments, it can be concluded that $\operatorname{Pr}\left(\mathcal{A}, M S_{(k)}\right)$ and $P_{e}\left(\mathcal{A}, M S_{(k)}\right)$ are the same for all sets $\mathcal{A}$ with the same cardinality. Hence, we have $P_{e}\left(A, M S_{(k)}\right)=$ $P_{e}\left(\mathcal{A}, M S_{(k)}\right)$ and $\operatorname{Pr}\left(A, M S_{(k)}\right)=\left(\begin{array}{l}N \\ A\end{array}\right) \cdot \operatorname{Pr}\left(\mathcal{A}, M S_{(k)}\right)$.

Sort the SNR of the source-relay channel in descending order $\gamma_{1,[1]}>\cdots>\gamma_{1,[N]}$. For simplicity of expression, denote $\gamma_{[i]}=\gamma_{1,[i]}$. The probability that exact $A$ relays $(A<N)$ and the $k$-th MS are selected is given by

$$
\begin{aligned}
& \operatorname{Pr}\left(A, M S_{(k)}\right) \\
& =\operatorname{Pr}\left(\left\{\Gamma_{(k)} \leq \min \left\{\sum_{i=1}^{A} \gamma_{2, i}, \gamma_{[A]}\right\}<\Gamma_{(k+1)}\right\} \bigcap\left\{\gamma_{[A+1]}<\Gamma_{(k)}\right\}\right),
\end{aligned}
$$

where $\Gamma_{(k)}=\Gamma\left(M S_{(k)}\right), k=1, \cdots, L$, and $\Gamma_{(L+1)}=+\infty$. Define $U_{A}=\min \left\{\sum_{i=1}^{A} \gamma_{2, i}, \gamma_{[A]}\right\}$ and $V_{A}=\gamma_{[A+1]}$. The joint probability density function (pdf) of $U_{A}$ and $V_{A}$ can be derived as in (10) at the top of the page. The proof of this result is given in Appendix A

Using the closed-form expression for $f(u, v)$ in (10), the expression for $\operatorname{Pr}\left(A, M S_{(k)}\right)$ can be written as follows:

$$
\begin{aligned}
& \operatorname{Pr}\left(A, M S_{(k)}\right) \\
&=\int_{0}^{\Gamma_{(k)}} \int_{\Gamma_{(k)}}^{\Gamma_{(k+1)}} f(u, v) d u d v \\
&=\frac{N !}{A !} \sum_{i=0}^{N-A} \frac{(-1)^{i} e^{-\tilde{\Gamma}_{(k)} i}}{i !(N-A-1) !} \cdot\left(e^{-(A+1) \tilde{\Gamma}_{(k)}} \sum_{j=0}^{A-1} \frac{\left(\tilde{\Gamma}_{(k)}\right)^{j}}{j !}\right. \\
&\left.-e^{-(A+1) \tilde{\Gamma}_{(k+1)}} \sum_{j=0}^{A-1} \frac{\left(\tilde{\Gamma}_{(k+1)}\right)^{j}}{j !}\right),
\end{aligned}
$$

where $\tilde{\Gamma}_{(i)}=\Gamma_{(i)} / \rho, i=1, \cdots, L$.

The $A=N$ case is treated separately from the $A<N$ case because no relays back off in this case. The probability that all relays and the $k$-th MS are selected can be derived as

$$
\operatorname{Pr}\left(N, M S_{(k)}\right)=e^{-(N+1) \tilde{\Gamma}_{(k)}} \sum_{j=0}^{N} \frac{\tilde{\Gamma}_{(k)}^{j}}{j !}-e^{-(N+1) \tilde{\Gamma}_{(k+1)}} \sum_{j=0}^{N} \frac{\tilde{\Gamma}_{(k+1)}^{j}}{j !} .
$$

The bound of the SER of the proposed scheme is given by,

$$
0<P_{e}\left(\mathcal{A}, M S_{(k)}\right) \leq S E R_{t g t} .
$$


Substituting (14)-(16) into (11) yields the upper bound and lower bound of the throughput for the proposed scheme,

$$
\zeta_{\text {upper }}=\sum_{k=1}^{L} \sum_{A=1}^{N} k \cdot \operatorname{Pr}\left(A, M S_{(k)}\right) \text {, }
$$

and

$$
\zeta_{\text {lower }}=\sum_{k=1}^{L}\left(k \cdot\left(1-S E R_{t g t}\right)^{L_{\text {bit }} / 2 k} \sum_{A=1}^{N} \operatorname{Pr}\left(A, M S_{(k)}\right)\right) .
$$

\section{B. Numerical Results}

Numerical results are provided in this subsection. In all results, the horizontal axis indicates the SNR of the source-relay and relay-destination channels without fading, and $L_{b i t}=1000$. The candidate modulation schemes used in the simulation are shown in TABLE \, while the SER target is $S E R_{t g t}=10^{-4}$.

TABLE I

CANDidate MSs and Corresponding SNR Threshold

\begin{tabular}{c|c}
\hline MS & Threshold \\
\hline No transmission & - \\
\hline QPSK & $11.80 \mathrm{~dB}$ \\
\hline 16QAM & $19.00 \mathrm{~dB}$ \\
\hline 64QAM & $25.32 \mathrm{~dB}$ \\
\hline 256QAM & $31.42 \mathrm{~dB}$ \\
\hline
\end{tabular}

In Fig. 4, the throughput in a network with 5 candidate relays is presented. It is observed that the proposed scheme increases the throughput relative to the link adaptation scheme without relay selection (with all candidate relays participating in the second step transmission) by over $0.5 \mathrm{bit} / \mathrm{s} / \mathrm{Hz}$ in the SNR range 10-35 dB. This is reasonable because relay selection can mitigate the risks that the channel is in deep fading by utilizing diversity. Also, the proposed scheme outperforms pure relay selection scheme with fixed MS. This is what should be expected from the proposed scheme because with the same average SNR at the destination, the proposed scheme can adaptively switch to a low or high spectral efficiency MS according to the channel realizations, so that the throughput is always maximized. Fig. 4 also indicates that the bounds derived in (17) and (18) are very close to the simulation results.

Fig. 5 shows the average SER of the proposed scheme in a 5-relay network. As is expected, the SER of the proposed scheme is always below the $S E R_{t g t}$. While a very low SER value may be not desirable from the point of the system level, maintaining it at an appropriate level, if not too high, is beneficial to maximize the system throughput. This is clear if we re-examine the spectral efficiency performance in Fig. 4 Although the SER of the proposed scheme is larger than relay selection with lower modulation rank in high SNRs, the throughput performance of the proposed scheme is improved.

\section{Signaling Comparison of the General Scheme (Section III- A) and Improved Scheme (Section III-B)}

In the general scheme, feedback signaling channel was prescheduled by scheduler in higher layer. Prior to scheduling,

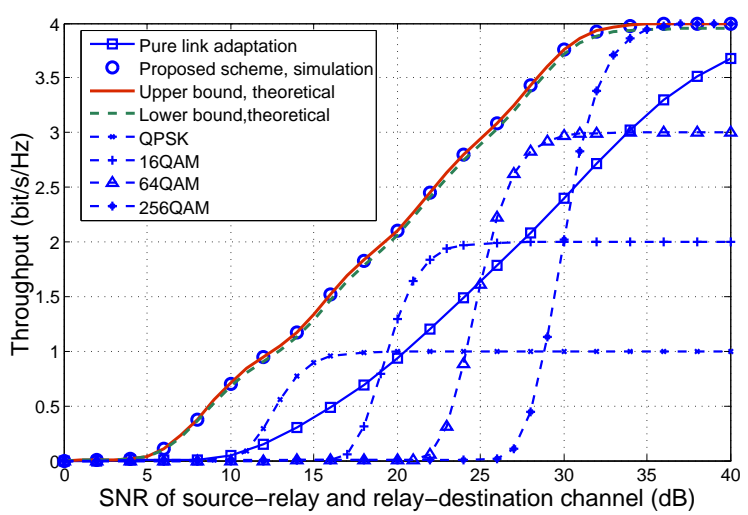

Fig. 4. Throughput performance of 5-relay networks. Six cases are studied: the proposed scheme, link adaptation with no relay selection, QPSK with relay selection, 16QAM with relay selection, 64QAM with relay selection and 256QAM with relay selection.

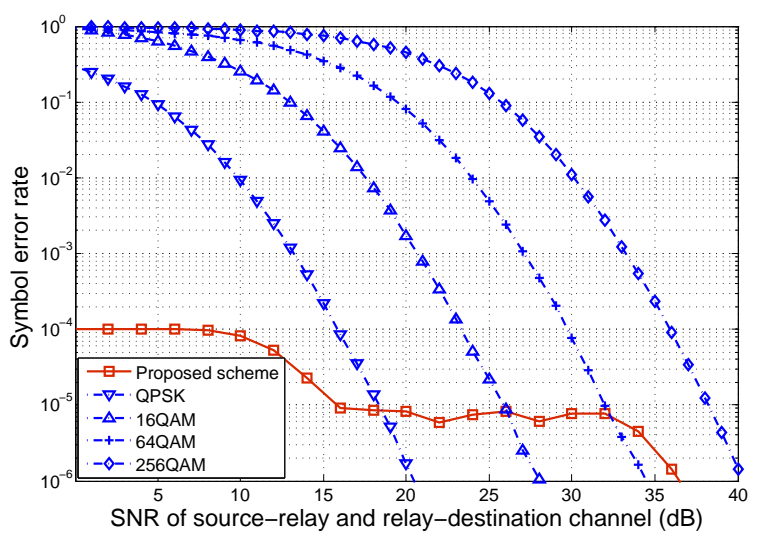

Fig. 5. Average SER of 5-relay networks. Five cases are studied: the proposed scheme, QPSK with relay selection, 16QAM with relay selection, 64QAM with relay selection and 256QAM with relay selection.

none of the nodes know what relays will be actually selected, which is based on instantaneous channel and packet detection error. So the signaling channel must be scheduled for all possible relays, both selected relays and not-selected relays. Since the feedbacks from destination to relays are in each relay's dedicated channel, the number of signaling bits is proportional to the number of relays.

However, in the improved scheme, signaling bits which indicate the link adaptation and relay selection results are transmitted in a broadcast channel. Here we calculate the number of signaling bits of the general scheme and proposed scheme for comparison, as shown in Table 2.

TABLE II

NUMBER OF SignALING BITS

\begin{tabular}{|l|l|l|}
\hline & TDD system & FDD system \\
\hline General & $N \times(\mathrm{MS}+1-\mathrm{bit}+\mathrm{Eg})+\mathrm{MS}$ & $N \times(\mathrm{MS}+1-\mathrm{bit}+\mathrm{BWI})+\mathrm{MS}$ \\
scheme & $=9 N+(N+1)\left\lceil\log _{2}(L)\right\rceil$ & $=17 N+(\bar{N}+1)\left\lceil\log _{2}(L)\right\rceil$ \\
\hline $\begin{array}{l}\text { Proposed } \\
\text { scheme }\end{array}$ & $\mathrm{MS}+\mathrm{Eg}=8+\left\lceil\log _{2}(L)\right\rceil$ & $\mathrm{MS}+N \times \mathrm{BWI}=$ \\
& & $16 N+\left\lceil\log _{2}(L)\right\rceil$ \\
\hline
\end{tabular}

In Table 2, $N$ is the number of relays, including both 
selected relays and not-selected relays. MS is the index of the selected MS, which is $\left\lceil\log _{2}(L)\right\rceil$ bits. The 1 bit in general scheme is the signaling bit that indicates each specific relay whether it is selected or not, and is assumed $1 \mathrm{bit}$. Eg is the summation of gaining factors. It is a real number and is assumed 8 bits. The BWI bits are used for both the two schemes in FDD system to indicate each relay the beamforming weight. They are assumed 16 bits, as they include the magnitude and phase. In the general scheme, the destination also needs to feedback MS to the source in the dedicated channel. By using the MS selection results to indicate the relay selection results, the bits used in general scheme for relay selection results feedback are saved, and the signaling overhead of relay selection and link adaptation is significantly reduced.

\section{Conclusion}

Motivated by the improvement in throughput and spectral efficiency afforded by link adaptation and relay selection, an approach for joint relay selection and link adaptation is proposed in this paper. Since the relay selection tends to select the links with higher capacity while the link adaptation makes efficient use of those links, the combination of relay selection and link adaptation can further provides synergistic gains. To reduce the signaling overhead of relay selection and link adaptation, a novel signaling mechanism is proposed, along with a simple and optimal selection algorithm. The bounds of the received SNR and the throughput is derived. Simulation results further confirms that the proposed scheme can improve the throughput and SER performance compared with pure link adaptation and relay selection schemes.

\section{ApPENDix A \\ Closed-Form EXPRESSION FOR $f(u, v)$}

Define $Z$ as $Z=\sum_{i=1}^{A} \gamma_{2, i}$, and the pdf of $Z$ is denoted by $g(z)$. Since $\gamma_{2, i}$ are independent exponential random variables, the Laplace transform, $\mathcal{L} g$, of $g_{Z}(z)$ is given by $\mathcal{L} g(s)=\frac{1}{(1+s)^{4}}$. Hence the pdf of $Z$ can be expressed as

$$
g(z)=\frac{z^{(A-1)} e^{-\frac{z}{\rho}}}{\rho^{A}(A-1) !}
$$

and the cumulative distribution function (cdf) of $Z$ is given by

$$
G_{Z}(z)=1-\sum_{i=0}^{A-1} \frac{z^{i} e^{-\frac{z}{\rho}}}{\rho^{i} i !}
$$

The joint pdf of $\gamma_{[A]}$ and $\gamma_{[A+1]}$ can be derived using the theory of "order statistics" [12] as

$$
\psi_{\gamma_{[A]}, \gamma_{[A+1]}}(x, y)=\frac{N ! e^{-\frac{y}{\rho}} e^{-\frac{A x}{\rho}}\left(1-e^{-\frac{y}{\rho}}\right)^{N-A-1}}{\rho^{2}(N-A-1) !(A-1) !}, \quad 0<y<x .
$$

By "order statistics", we also have

$$
\psi_{\gamma_{[A+1]}}(y)=\frac{N ! e^{-\frac{(A+1) y}{\rho}}\left(1-e^{-\frac{y}{\rho}}\right)^{N-A-1}}{\rho(N-A-1) ! A !}, \quad y>0,
$$

where $\psi_{\gamma_{[A+1]}}(y)$ is the pdf of $\gamma_{[A+1]}$. Thus, the joint cdf of $U_{A}$ and $V_{A}$ can be expressed as

$$
\begin{gathered}
F(u, v)=\left\{\begin{array}{cc}
\operatorname{Pr}\left(Z>u, \gamma_{[A]}<u, \gamma_{[A+1]}<v\right) \\
+\operatorname{Pr}\left(Z<u, \gamma_{[A+1]}<v\right), & 0<v<u \\
\operatorname{Pr}\left(Z<u, \gamma_{[A+1]}<v\right), & 0<u<v
\end{array}\right. \\
=\left\{\begin{array}{cc}
(1-G(u)) \Psi(u, v)+G(u) \Psi_{\gamma_{[A+1]}}(v), & 0<v<u \\
G(u) \Psi_{\gamma_{[A+1]}}(v), & 0<u<v,
\end{array}\right.
\end{gathered}
$$

where $\Psi(u, v)$ denotes the joint cdf of $\gamma_{[A]}$ and $\gamma_{[A+1]}$. Thus the joint pdf of $U_{A}$ and $V_{A}$ can be derived as

$$
f(u, v)=\left\{\begin{array}{cl}
g(u) \psi_{\gamma_{[A+1]}}(v)-G(u) \psi(u, v) & \\
-g(u) \frac{\partial \Psi(u, v)}{\partial v}+\psi(u, v), & 0<v<u \\
g(u) \psi_{\gamma_{[A+1]}}(v), & 0<u<v,
\end{array}\right.
$$

where

$$
\frac{\partial \Psi(u, v)}{\partial v}=\frac{N ! e^{-\frac{v}{\rho}}\left(1-e^{-\frac{v}{\rho}}\right)^{N-A-1}\left(1-e^{-\frac{A u}{\rho}}\right)}{\rho(N-A-1) ! A !} .
$$

The closed-form expression for $f(u, v)$ can be derived by substituting (19)-(22) and (25)into (24).

\section{REFERENCES}

[1] J. N. Laneman, David. N. C. Tse, and G. W. Wornell, "Cooperative Diversity in Wireless Networks: Efficient Protocols and Outage Behavior," IEEE Trans. Inform. Theory, vol. 50, no.12, pp. 3062-3080, Dec. 2004

[2] R. Madan, N. B. Mehta, A. F. Molisch, and J. Zhang, "EnergyEfficient Cooperative Relaying Over Fading Channels With Simple Relay Selection," IEEE Trans. Wireless Commun., vol. 7, no. 8, pp. 3013-3025, Aug. 2008.

[3] A. Goldsmith and S. G. Chua, "Adaptive coded modulation for fading channels," IEEE Trans. Commun., vol. 46, no. 5, pp. 595-602, May 1998.

[4] Z. Lin, E. Erkip, and M. Ghosh, "Rate adaptation for cooperative systems," IEEE GlobeCom, San Francisco, USA, Nov. 2006, pp. 1-5.

[5] Y. Jing, and H. Jafarkhani, "Single and multiple relay selection schemes and their achievable diversity orders," IEEE Trans. Wireless Commun. vol. 8, no. 3, pp. 1414-1423, Mar. 2009.

[6] A. Bletsas, A. Khisti, D. P. Reed, and A. Lippman, "A simple cooperative diversity method based on network path selection," IEEE J. Select. Areas Commun., vol. 24, no. 3, pp. 659-672, Mar. 2006.

[7] Y. Jing and H. Jafakhani, "Network beamforming using relays with perfect channel information," IEEE Trans. Inf. Theory, vol. 55, no. 6 , pp. 2499-2517, Jun. 2009.

[8] R. Mudumbai, D. R. Brown III, U. Madhow, and H. V. Poor, "Distributed transmit beamforming: challenges and recent progress," IEEE Commun. Mag., vol. 47, no. 2, pp. 102-110, Feb. 2009.

[9] E. Koyuncu, Y. Jing, and H. Jafarkhani, "Distributed beamforming in wireless relay networks with quantized feedback," IEEE J. Select. Areas Commun., vol. 26, no. 8, pp. 1429-1439, Oct. 2008.

[10] L. Wang, C. Zhang, J. Zhang, and G. Wei, "Distributed beamforming with limited feedback in regenerative cooperative networks," in IEEE WiCom, Beijing, China, Sep. 2009, pp. 1-4.

[11] M. R. Souryal and N. Moayeri, "Joint Rate Adaptation and ChannelAdaptive Relaying in 802.11 Ad Hoc Networks," in IEEE Military Communications Conference, Washington D.C., USA, Oct. 2006, pp. $1-8$.

[12] P. J. Bickel and K. Doksum, Mathematical Statistics: Basic Ideas and Selected Topics, first ed. Oakland, CA: Holden-Day, 1977. 